\title{
The sixth base and counting
}

Enzymatic glycosylation and oxidation of methylated DNA together with highthroughput sequencing allows genomewide base-resolution of 5-hydroxymethyl cytosine distribution.

5 -Hydroxymethyl cytosine $(5-\mathrm{hmC})$ is increasingly being recognized as a base in its own right rather than an intermediate of methyl cytosine $(5-\mathrm{mC})$ on its way to demethylation. 5-hmC, touted as the sixth base, is produced by ten-eleven translocation (TET) enzymes as they oxidize 5-mC.

Whereas the role of 5-mC in epigenetic regulation is undisputed, 5 -hmC's function is less clear, but it putatively includes the regulation of gene expression and DNA demethylation, particularly in embryonic stem cells and mammalian brain cells, where 5-hmC levels are high.

To understand what 5 -hmC is doing, one needs to know where it is. In the last 3 years, researchers have developed various tools and methods to enrich 5-hmC-bearing regions. In 2010, Chuang He from the University of Chicago used glycosyltransferase to tag 5 -hmC with an azido glucose analog to which he appended biotin via click chemistry, making for an easy pulldown.

All of these enrichment methods led to valuable insights about the distribution of 5-hmC, but, says He, they all have a substantial drawback in their inability to assess abundance. "A genome-wide look at relative abundance is very important to get a real view of the biological function of 5-hmC," he says.

Such a genome-wide look is only possible through sequencing. But although sequencing of 5-mC is done by bisulfite conversion of unmethylated Cs to Us, bisulfite treatment does not distinguish between $5-\mathrm{mC}$ and 5-hmC, neither is converted to $\mathrm{U}$ and both are read as $\mathrm{C}$ in bisulfite sequencing. Thus He needed a method to distinguish the fifth from the sixth base.

His team revisited the glucose-tagging strategy and found that glycosyl-5-hmC is protected from further oxidation, whereas unprotected 5-hmC gets oxidized to formylcytosine (fC) and then to carboxylcytosine (caC). After protecting 5 -hmC in human embryonic stem cells, the researchers simply let the TET enzyme do its job overnight of converting most 5-mCs to caCs, which are read as Ts after bisulfite conversion. Glucoseprotected 5-hmCs, on the other hand, were

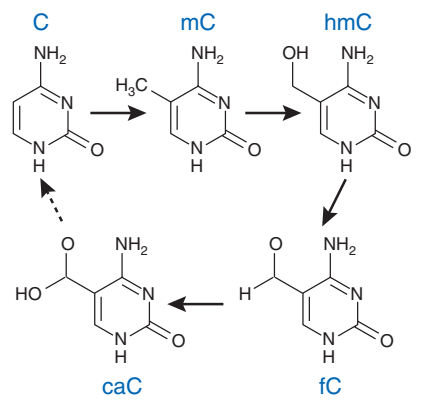

Enzymatic steps of cytosine methylation and demethylation.

still being read as Cs, thus providing a direct readout of their positions.

$\mathrm{He}$ and his colleagues Bing Ren at the University of California, San Diego, and Peng Jin at Emory University found almost half of all abundant 5-hmC sites to be in distal regulatory regions, areas with low overall methylation levels. The researchers also found 5-hmC to be enriched around transcription factor (TF) binding sites but depleted in the binding motif itself. When asked what this might say about the function of 5-hmC, He is quick to point out that these findings need further scrutiny, but he nonetheless speculates, "It is my sense that specific complexes, including the TF, may recruit TET1 proteins, which demethylate the neighborhood [and may thereby influence gene expression]. This agrees very well with the correlation of 5-hmC with regulatory elements."

What is clear is that the distribution of 5 -hmC in human embryonic stem cells is not random, which would be expected if it were simply a byproduct of demethylation, but instead is generated and maintained at specific loci involved in the regulation of gene expression.

And if 5-hmC has specific roles, this raises the question of whether the downstream oxidation products $5-\mathrm{fC}$ and $5-\mathrm{caC}$ do as well. Is there a 'methylation code', a carefully orchestrated combination of 5-mC, 5-hmC, 5-fC and $5-\mathrm{caC}$, to fine tune cellular function? Methods specifically geared toward examining each of these nucleotides are needed to answer this question.

Nicole Rusk

\section{RESEARCH PAPERS}

Yu, M. et al. Base-resolution analysis of 5 -hydroxymethylcytosine in the mammalian genome. Cell 149, 1368-1380 (2012). 